\title{
Co-operating with Municipal Partners on Indicator Identification and Data Acquisition
}

\author{
Oliver Ullrich, Manfred Bogen, Daniel Lückerath, Erich Rome \\ Fraunhofer Institute for Intelligent Analysis and Information Systems IAIS, Schloss Birlinghoven, \\ 53757 Sankt Augustin, Germany; oliver.ullrich@iais.fraunhofer.de
}

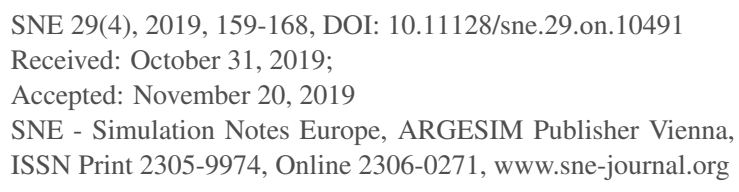

SNE - Simulation Notes Europe, ARGESIM Publisher Vienna, ISSN Print 2305-9974, Online 2306-0271, www.sne-journal.org

Abstract. Data acquisition is a significant part of the simulation modeling process. It can be a challenge, especially in larger projects with many participants and stakeholders from different professions: They all bring their individual understanding of data, its availability, formats, and quality, with their respective points of view strongly dependent on their own professional backgrounds. This paper presents a modular process for indicator identification and data acquisition in the context of modeling projects, specifically aimed at researchers or practitioners working with municipal partners, i.e. representatives from city councils, urban planners and administrations, or infrastructure providers. The process consists of five steps: indicator selection, suitability check, data gathering, data quality check, and data management. To further facilitate a clearer understanding of the endeavor, the process defines necessary input and to be expected output artifacts. In addition to presenting the process, the paper describes its examplary execution with partners from the City of Bilbao, Spain, and offers a selection of discovered challenges and lessons learned.

\section{Introduction}

In complex multi-disciplinary projects with modeling and simulation components, the acquisition of highquality data can be a significant challenge. Often, data owners are not modeling experts and without further guidance lack the experience to understand what data is necessary in what form.
Especially in projects where data owners are representatives from city administrations or municipal infrastructure providers, these public servants often have the detailed local knowledge that is needed to develop an effective and efficient model. However, their typically relative low level of experience with the modeling and simulation process means that they can benefit from some extra guidance on the more complex steps of the identification of feasible and valid indicators that quantitatively describe the desired characteristics, and the corresponding acquisition and preprocessing of the necessary data.

This paper describes a process designed to assist modelers in projects with municipal stakeholders with the identification of feasible and expressive indicators and the corresponding acquisition and preparation of the necessary data. The process addresses typical stumbling stones and road blocks, and has been validated in a number of case studies in co-operation with the municipalities of Bilbao, Spain, Greater Manchester, UK, Paris, France, and Bratislava, Slovakia.

The described process has been first developed as part of "Impact and Vulnerability Analysis of Vital Infrastructures and Built-up Areas - IVAVIA" (see [24]), a standardized method for the assessment of climate change-related risks and vulnerabilities in cities and urban environments, based on the well-established approach by the German Federal Ministry for Economic Cooperation and Development (see [12]). IVAVIA was conceived as part of the EU-H2020 project "RESIN - Climate Resilient Cities and Infrastructures" (see [23]) and is further developed in the EU-H2020 project "ARCH - Advancing Resilience of Historic Areas Against Climate-related and Other Hazards" (see [2]).

The paper continues with a short introduction on the background of indicator identification and data acquisition in co-operation with municipal partners (see Section 1) and then presents a systematic process designed to help municipalities with these steps (see Section 2). 
The paper then describes the application of the process in the context of modeling climate change-related risks and vulnerabilities for the city of Bilbao, Spain, including the encountered challenges (see Section 3). It then elaborates on some lessons learned in the exemplary execution of the process (see Section 4), and concludes with a short summary and an outlook on the next research steps (see Section 5).

\section{Background}

An "indicator" is a general concept in statistics, with specialisations in other disciplines (see [25]). In simulation (input) modeling, indicators provide information about states or conditions that are not directly measurable as part of the examined system; they may relate directly or indirectly to the element they are intended to measure. When used in general modeling, the indicator is a variable that contributes to describing the properties of a system. In this context "data" is seen as the specific values of indicators, changing over time or space.

Literature reviews show that data gathering and input modeling is an active field in simulation modeling (see [8]). While more technical introductionary texts on simulation mention data gathering only in passing (see [3] and [30]), Carson (see [5]) describes in his overview on the modeling and simulation process often encountered challenges with data collection, cleansing and analysis: data might not be available or not of the desired quality, unforeseen effort is necessary to clean up databases and files, with clients often not knowing what data they own and whether it is acurate.

A number of authors reported progress at solving the identified issues: Bengtsson et al. (see [4]) introduce processes and tools for examining well-structured systems in the aerospace industry, Perara and Liyanage (see [20]) apply a structured process for batch manufacturing environments, while Skoogh et al. (see [28]) aim to support data management in comparable situations. All these proposals assume well-structured environments with stakeholders from manufacturing or industry that share a certain degree of understanding of computer-based modeling.

Especially in large co-creation projects with many stakeholders who do not share that background, indicator identification and data collection and preparation can necessitate even more significant efforts, with literature suggesting that approximately $30 \%$ (see [27]) or more (see [20]) of effort in simulation projects are caused by it. In case local or municipal systems or infrastructure components are subject of the examination, usually at least some of these partners are not modeling experts but representatives of the city administration or local infrastructure providers who lack experience with the modeling and simulation process.

Here, co-creation methods might be useful tools. In co-creation or co-management driven projects methods and models are designed, developed, and tested in close feedback loops with end-users instead of applying a traditional waterfall approach that involves nonexperts only at the beginning of the process, in the requirements engineering phase, and at the very end, in the application phase. In large modeling projects with municipal stakeholders applying such techniques helps to avoid the trap of being perceived as being purely science-driven (see [7]) and ensures that project results have high practical applicability and usability values (see e.g. [6], [13], and [22]). Municipal stakeholders are reported to perceive co-creation projects as more perceptive to local issues, with an improved connection to their existing agendas (see [18] and [29]). Especially in the context of the co-management of environmental change and the adaptation to climate change in projects involving municipal partners, co-creation processes have been observed to facilitate the implementation of effective multi-level measures (see [1], [21], and [31]).

\section{A Process for Indicator Identification and Data Acquisition}

\subsection{Input}

A planned and structured approach to identify indicators and acquire data in co-operation with municipal partners requires some necessary input. That includes documented results of earlier modeling stages and informal local knowledge, but not large amounts of formalized data.

Main input documents are conceptual models, yet without qualitative attributes, that the modeler will have prepared during earlier stages. These conceptual models (see [5]) might have been prepared using one or more of the usual modeling techniques, resulting in a number of event-driven process chains (see [26]), UML activity diagrams (see [9]), impact chain diagrams (see [14]), or other artifacts. 
The need for informal local knowledge is a bit harder to grasp. Here, that knowledge would not only include a deeper understanding of the municipal systems or infrastructure components to be modeled, but also the knowledge of what stakeholders have access to the required information, and whether it is available at the necessary levels of functional, spatial, and temporal resolution. The authors found it helpful to identify at an early stage a project data champion inside the local administration who has informal access to colleagues and representatives from local administration departments and other stakeholders. At this stage, it is important to include all relevant stakeholder groups with the project, and to facilitate a clear understanding of the aims and scope, and the knowledge and data necessary for a successful conclusion. Again, an insider can help to identify all relevant stakeholders.

\subsection{Steps}

The process consists of five steps to be executed by the modeler in co-creation with municipal stakeholders (see Figure 1).

Select Indicators. In modeling, indicators provide information about states or conditions that are not directly measurable; they may relate directly or indirectly to the element they are intended to represent. Examples of indicators in municipal contexts include:

- the geo-referenced distribution of education levels as an indicator for the degree of receptiveness for complex information on climate change-related issues,

- the geo-referenced distribution of age as an indicator for the vulnerability to heat waves, or

- the distribution of public service interruptions as an indicator for the resilience of infrastructure components.

The values of the selected indicators might later be aggregated with additional components to form a composite indicator. Therefore, the stakeholders need to select at least one indicator for each system state or condition to be represented in the model.

How to start identifying indicators? Established indicator directories can be a great help with identifying suitable indicators. For modeling climate changerelated issues in municipal contexts, such directories can be found e.g. in the annex of the Vulnerability Sourcebook (see [12]), the Covenant of Mayors for Climate and Energy Reporting Guidelines (see [17]) or the indicator database of the EU FP7 project "MOVE Methods for the Improvement of Vulnerability Assessment in Europe" (see [16]). However, because indicators are only useful if the relevant data is available for a local context, a modeler should already start considering existing and necessary data when compiling a set of potential indicators: The best indicator is inoperable if there is no feasible way of acquiring the necessary data. The whole indicator selection and data acquisition activity is an iterative and potentially time-consuming process: identifying an indicator, checking its suitability, gathering data, reformulating the indicator if no suitable data can be found to substantiate it, checking data quality and finding alternative sources where necessary. Subsequently, the described steps should not be considered as isolated parts of a sequential process, but rather as different views of an iterative process.

It is important and advisable to include local expertise, e.g. from technicians and other experts working within the urban area or municipality, who know what data is available. In addition to using the already mentioned indicator directories, it might be worth to examine previous studies for the examined region or city in order to reduce the number of potential indicators for which to check data availability. Finally, it is important to find a consensus between the involved experts and stakeholders on what indicators can and should be employed as part of the simulation modeling project.

Developing a preliminary list of potential indicators creates a significant overhead when it is done the first time. However, a thorough documentated selection process will significantly ease the effort of indicator examination in further iterations of the step and in later modeling projects with comprehensive data demands.

The outputs of this step are:

- Preliminary list and documentation of all potential indicators

Check for Suitability. After potential indicators have been identified, they need to be assessed for their suitability towards the purpose of the model: 
Steps

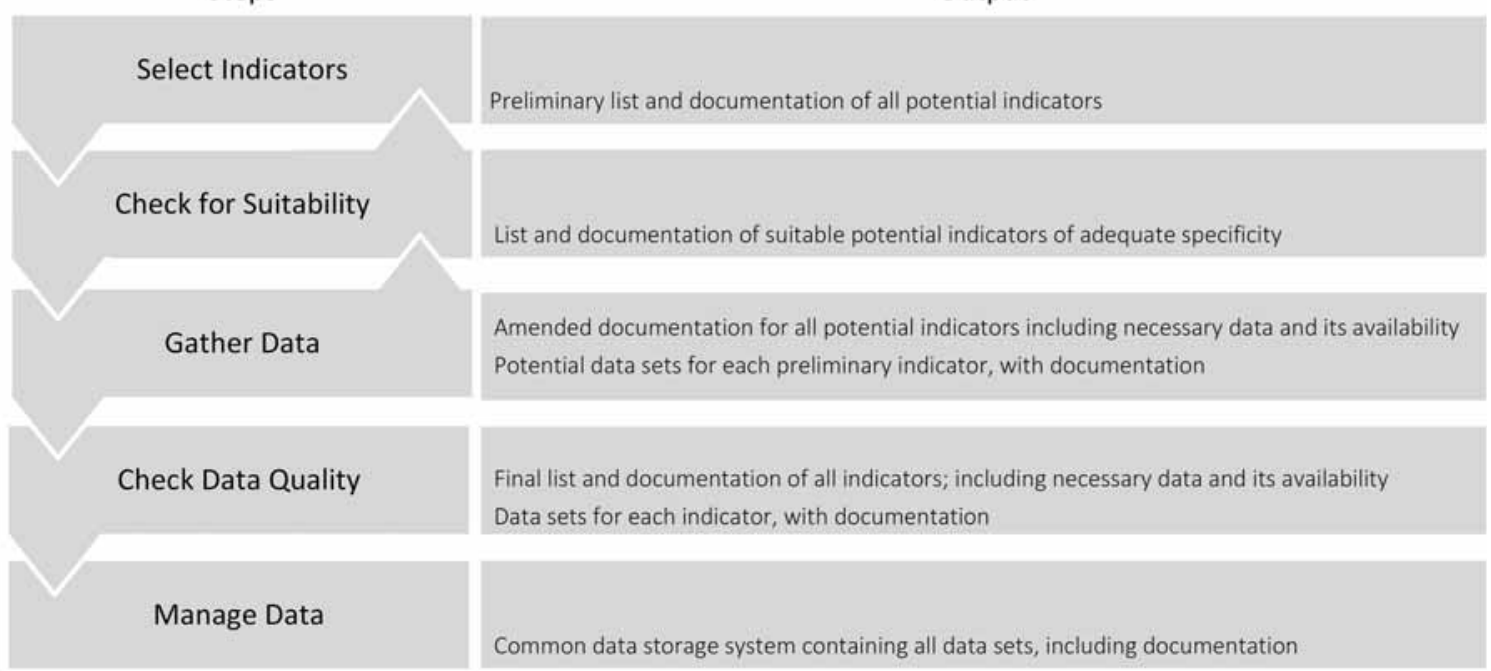

Figure 1: Process steps for indicator identification and data acquisition.

- Are they valid and relevant, i.e. do they represent well the elements to be modelled?

- Are they reliable and credible and allow for data acquisition and measurement in the future?

- Do they have a precise and documented meaning, i.e. do stakeholders agree on what the indicators are measuring in the context of the model?

- Are they clear in their direction, i.e. is an increase in value unambiguously positive or negative?

- Are they practical and affordable, i.e. do they come from accessible data sources?

In an urban or municipal context, two dimensions of appropriateness are often especially relevant:

- Geographical coverage. The identified indicators should cover the full extent of the study area (e.g. the whole urban city or district) and have an appropriate resolution (e.g. population data on a district level).

- Temporal coverage and time frame. Depending on the different indicators identified and whether or not historical periods need to be modeled, archival records and/or future projections need to be available. Additionally, that data should cover the same temporal interval with the same resolution, e.g. daily precipitation and traffic volume for a whole year.
Indicators that are not suitable should be disregarded and their entry in the list of potential indicators amended with a corresponding comment to allow for the reconstruction of the process by non-participating colleagues later on. Should the pruning of the potential indicator list result in elements of the preliminary qualitative model without indicators, the modeler may need to go back to the corresponding step in the process, try to find new indicators and re-iterate the specificity check.

The output of this step is:

- List and documentation of suitable potential indicators of adequate specificity

Gather Data. The process of gathering data for a simulation model of an urban or municipal system can range from extremely simple to highly challenging. It may suffice to download available census data or GIS maps from open access websites. In some cases, time and resources permitting, surveys need to be conducted or large, complex data sets, like satellite images, need to be processed - which might require specialist skills.

What kind of data is needed? There is no standard solution for all possible models. While some indicators will most likely require physically measured or modeled (historical) data, indicators representing social or demographic issues will often require survey and census data or data from expert judgement. However, the 
most important decision criteria for what data is needed are the study area (e.g. the city as a whole or individual city districts), the desired geographical resolution of the model (e.g. districts, neighbourhoods, smaller areas within neighbourhoods), and the output type (e.g. maps, diagrams, tables) and level of detail (e.g. result validation via visual analysis of the employed data sets).

E.g., if the model represents individual municipal districts, data should at least have a district level resolution (e.g. population data for complete districts or individual households, which then can be assigned to corresponding districts). Data at a lower resolution (e.g. population data for the whole city) usually cannot be disaggregated without loss of validity. As another example, if the simulation results should be represented visually as a map, corresponding geographic data (e.g. a shape file) is needed. Similarly, if detailed (visual) validation of results seems necessary or helpful, the corresponding data needs to be provided in an appropriate format.

Does the data already exist? Based on the information gathered as input for the process (see Section 2.1) a modeler should be able to identify a first set of relevant organizations, facilities, and experts on a local, regional, national, or international level that may be able to provide the necessary data. The huge number of institutions and experts that might need to be contacted can make this one of the most time-consuming steps of the process, especially as follow-up negotiation is often required. Depending on the selected indicators, statistical offices, meteorological authorities, and different national and local government departments might need to be approached. National Spatial Data Infrastructures (NSDI) are another key entry point for data acquisition. NSDIs have been established in many countries and will ideally offer standardised data, even where it is sourced from multiple institutions (see [12]).

When gathering data and contacting institutions and experts, a modeler has to be aware of intellectual property rights levels and sharing policies that may be in place; formal agreements with the respective rights holders could be required.

What resources can be committed to generate data? If data is not available or of insufficient quality, data might need to be collected by the modelers themselves.
In that case, careful assessment of the required costs and expertise needed for the data collection is needed.

Regarding climate-related municipal data, the Vulnerability Sourcebook gives some useful hints (see [12], p. 96-100):

"For meaningful results, observation of biophysical indicators such as precipitation, temperature and run-off must be made over long periods - often over decades. The time and money required for this means it is almost certainly unfeasible for [a modeler to gather this data yourself]. Luckily, however, most countries can provide such data [, e.g. via climate service provides such as hydrometeorological offices]. If you require highly localised data, expert judgement may be a worthwhile alternative."

"Data for socio-economic indicators such as average household income, average size of household and livelihood strategies can be captured in surveys. The time and money required depend largely on the sample size. A representative survey may cover a whole country, or just a few communities. At the sub-national level, surveys can be an effective means of gathering information not captured by national institutions, such as perceptions around climate and environmental change. [In large cities, socio-economic survey data often might have already been collected by a statistics or data department.] Be sure to involve a local expert who can help in drafting the survey, selecting a representative sample and analysing the resulting data."

"Modelled data are both time- and resourceintensive and usually require measured data as input. [...] For meaningful results, you will need to ensure that you can call on the required modelling skills." Often, already existing sub-models for fluvial/pluvial flooding, temperature, and urban heat isles may be available on a regional or national level.

"Where time and financial resources are limited, expert judgement can be a good, fast way of quantifying indicators that cannot otherwise be assessed. This is most often the case at a very local level - [e.g. when gathering information on the municipal sewer system from city technicians] - which is rarely covered by detailed statistical data, and where the climatic and hydrological characteristics are too specific to be captured by modelling. This local knowledge - captured using participative methods as well as scoring and ranking - can be used to either complement or replace surveys." 
Given the extent of the data gathering process and its inherent iterative nature, it is perfectly acceptable to conduct the process in an iterative fashion. That is, instead of gathering all potentially necessary data before starting to work on the model itself, it may be more practicable to start with a minimum amount of data necessary. While partial results may lack validity, they may help you when communicating with stakeholders and to secure stronger commitment.

Outputs of this step are:

- Amended documentation for all potential indicators that are specific enough, including what kind of data is needed to quantify the indicator and if it already exists or not

- Potential data sets for each preliminary indicator, with documentation

Check Data Quality. The validity of the output of a simulation model strongly depends on the quality of the input data. Subsequently, a modeler needs to assure that the data gathered is of sufficient quality. Such a check may reveal major issues with the data quality, which may necessitate to repeat at least part of the process. To avoid this, the quality criteria below should already be considered during the data collection. The following paragraphs describe some of the common issues with data quality, both in form and content.

Data format. The collected data can be provided in different formats. To enable easy handling, data should be provided in a well-structured, preferably digital file format that is easy to interpret, does not need manual reformatting, and can potentially be handled by colleagues without the need for specific (software) tools. Literature provides a (non-exhaustive) list of digital file formats that comply with these requirements (see [25]).

Temporal and geographical coverage. The geographical and temporal coverage of different data sources may vary. Thus, the stakeholders need to determine whether different data sources can be combined and compared or not.

In general, data should have the same geographical and temporal coverage. Additionally, data should ideally be as recent as possible and have, or at least be convertible to, the same temporal resolution (e.g. hourly precipitation and traffic volume from June 2017).
However, some differences in data timeliness and temporal coverage may be tolerable. This is especially true if data changes comparatively slowly, e.g. age distribution of citizens or other census data. Additionally, if indicators are independent from one another using data sources with different temporal coverage and resolution may be acceptable. E.g., the amount of green infrastructure in a city and the annual household income will most likely not be related and subsequently a difference in temporal coverage may not be significant.

Another problem may arise if geographical data uses different coordinate systems and projections. This is especially frequent when working on cross-border regions. In order to combine and compare different data sources, a common geographic reference system needs to be employed, such as the Universal Transverse Mercator coordinate system.

In any case, having some data is preferable to having no data at all. If no other alternative to using poor data is available, use it - but be aware that the results of the modeling process may be less reliable or may even be misleading.

Missing values or "outliers". Missing values (e.g. regions omitted from geographical data) can be problematic for simulation modeling. Smaller gaps can be closed with interpolation, i.e. finding existing data nearest to the gaps (in space, time, or function) most likely matching the missing data, or by using the average value as a replacement, if no other data is available. The Handbook on Constructing Composite Indicators by the OECD (see [19]) goes into more detail about missing values and how to deal with them.

"Outliers" on the other hand are values that are so far outside the expected range of the data that they may indicate an error in the capturing or calculation method. While these cases do happen, e.g. from technically unreliable measureing equipment, unexpected data should not be disqualified easily. Are these data points really technical mishaps, or are they a sign that parts of the system to be modeled have not been understood sufficiently?

Outputs are:

- Final list and documentation of all indicators; including what kind of data is needed to quantify the indicator and if it already exists or not

- Data sets for each indicator, with documentation, including physical dimensions 
Manage Data. To avoid data loss and lower the risk of data redundancy, all participating stakeholders should store the gathered data sets in a common data storage system. This may range from a simple collection of data files in a set of folders to a more complex database system. Depending on the system employed, they may need to transform the different data sets into a common data format, utilising export and transformation routines from multiple software products. Once such a data storage system is available, its contents can be used for other projects.

In a project with multiple partners and stakeholders it may be necessary to ensure that they can all access the different datasets and work with the same format. Additionally, responsibilities for database management and maintenance might need to be assigned to a "caretaker" or an external service provider. Finally, sensitive data has to be stored in a secure way, only accessible by the appropriate users. What exactly is seen as "sensitive data" is dependent on the country or even region of origin. A good start would be to check the EU General Data Protection Regulation (see [10]) and its national implementation, as well as additional national and regional legislation.

At this point all the gathered data should be documented precisely and comprehensively, allowing all internal and external colleagues, partners, and stakeholders to understand the format and meaning of the data, as well as access rights. Insufficient knowledge about the data can lead to unnecessary duplicate effort from colleagues, data loss, missing results, or a lack in transparency and credibility.

Output of this step is:

- Common data storage system containing all data sets, including documentation, esp. on format and meaning, access rights

\subsection{Output}

After the successful conclusion of the process steps a modeler has gained a set of documents, including files of actual data, as well as informal knowledge on the to be examined system and its stakeholders. These documents include a final list and documentation of all necessary indicators and the attributes of the system they represent, and a data storage system containing all data sets, including documentation, in the required formats, quality, and granularity.

In addition, a plethora of informal knowledge will have been gained: On one hand, that includes knowledge the modeler gains on the examined system and the municipal stakeholders. The modeler understands which stakeholder has expertise in what specific domain and, should that turn out to be necessary, can provide additional information on a sub-system. On the other hand, the co-operation process will have typically facilitated a better understanding of the modeling and simulation project for the local stakeholders. In the course of the workshops with municipal stakeholders they executed (see Section 3), the authors found that in the best case the project may gain dedicated champions inside the municipal administration.

\section{Example}

The described process was executed by the authors and representatives from a number of different administrative departments of the City of Bilbao, Spain, as well as other research partners in the context of modeling the impacts of global warming on local residents and infrastructure.

The project started out with an impact chain workshop attended by representatives from different departments where the municipality decided to focus on modeling three cause-impact relationships they thought especially relevant for their city: the impact of extreme precipitation on city traffic infrastructure, the impact of heat waves on public health, and the impact of flooding in built-up areas.

It was clear early in the project that to successfully model these relationships in a meaningful way substantial amounts of data would be necessary that up until then were kept in separate information silos in the different branches of the municipal government. It was thus decided to test the described process in the Bilbao context.

Additional details on the assessment conducted in the Bilbao urban context can be found in [15].

\subsection{Identifying Indicators and Acquiring Data for Bilbao}

The process of gathering data for the quantitative modeling stages was initiated in parallel to the finalization of the qualitative impact chains. After the immediate start of indicator selection, the project partners began to check whether corresponding sets of data were available and, over the following months, sought to obtain 
them by establishing contact with different departments within the municipality. As an intermediate result, the partners prepared a first overview of the data found so far for the selected model components and provided the data in differing formats.

The gathered data allowed the authors to start testing and refining the quantitative models and provide intermediate results and visual aids, e.g. maps and charts. Based on discussions of these some of the planned indicators were rejected. That included indicators that were found to be not suitable to measure the desired attribute as initially hoped for by the municipality, such as measureing road accessibility by the number of available CCTV cameras. It was also found that new or additional data had to be provided than initially envisioned by the city administration, e.g. position and specific characteristics of historical public buildings and position of emergency access ways to underground infrastructure components especially prone to flooding.

Data provided in shape file format was processed using a geographic information system software, e.g. to calculate the surface area of green infrastructure or population density per neighbourhood. Data provided in other formats was either converted to shape files (e.g. maps only available as PDF files), used to create new shape files for more complex calculations (e.g. information about coverage area of emergency services was used to create shape files representing the influence radius and subsequently the percentage of population covered by emergency services per neighbourhood), or used directly for indicator calculation (e.g. for capacity of storm tanks).

Approximately nine months into the project the partners collectively decided on the normalization, weighting, and aggregation methods for the selected data at the neighbourhood scale. At the end of that month, an inperson meeting was held in Bilbao between the modelers and the stakeholders to go over the results. The basic model was completed and presented at a workshop organized by the municipality approximately a year after the start of the city case. Then, the municipal stakeholders decided that the spatial resolution was too coarse for the envisioned use of the model. Therefore, the partners tessellated the available data at a finer spatial resolution: a 25 by 25 meter grid, yielding some 66,000 cells for the entire surface of Bilbao. While this resolution allowed for the examination of single city blocks and even individual buildings, the drawback was the labourintensiveness of preparing the data that way.

\subsection{Encountered Challenges}

During the course of the described (and other) test cases a number of common challenges and difficulties were encountered, mainly regarding data availability and quality. These include:

- Data sets that employ unwieldy data formats. E.g., a map may only be available as a PDF file without scale, complicating the calculation of surface area and circumference.

- Data sets describing different attributes of the same geographic element may employ different representations of the geographic element, e.g. two shape files of a geographic information system, one describing road elevation and one describing traffic intensities, may show differences in the geographic representation of the underlying road system.

- Data sets with different spatial and/or temporal resolutions. If multiple data sets have to be combined to calculate an indicator, they should have the same spatial and temporal resolution, i.e. they should cover the same area with the same detail over the same time interval. In some cases, it is possible to alleviate differences in resolution, e.g. data from a data set with a very high resolution may be aggregated to fit lower resolution data sets. However, disaggregating data from a low-resolution data set is usually not possible without loss of validity.

- Using the collected data at a later modeling stage may also reveal that a chosen indicator is, against expectations, not suitable to measure the desired phenomenon, e.g. the number of traffic cameras cannot be employed to measure the accessibility of a certain road segment. In this case, it is necessary to replace the indicator with a more suitable one, and to redo the process at least partially.

\section{Lessons Learned}

The described co-operation process has been tested in the context of four different city cases (see [15]). During that period a number of lessons have been learned both regarding the co-creation process in general and more technical and data quality issues.

Some general lessons from these assessments are: access to local knowledge is necessary, the process cannot be successfully be implemented by external experts 
only, the process will not be executed successfully without personal contact, face-to-face meetings with local end-users and external experts are necessary, it is important to ensure a common understanding regarding aims, scope, roles, required data, and terminology, it should be made clear from the beginning that the availability of sufficient resources is necessary, offering a reference set of indicators to the local experts can be beneficial and improves the general quality of results, and acquiring necessary data takes much longer than you think - always! A set of requirements concerning data quality and technical issues were identified, all of which have to be fulfilled to ensure the successful execution of the modeling process further down the road: data has to be complete according to specification; data and documentation have to be made available in a language understood both by local domain experts and the modelers; the semantics have to be clear; the spatial and temporal resolution has to be adequate; data formats have to be standardized and well documented; contradictions have to be resolvable or-preferably-not present; and files must not be defective.

\section{Conclusion}

This paper introduced a process for indicator identification and data acquisition in co-operation with municipal project partners. The process consists of five steps: indicator selection, suitability check, data gathering, data quality check, and data management, and defines the necessary input and the to be expected output documents. As an example, the execution of the process with partners from the City of Bilbao, Spain, was described, and discovered challenges and lessons learned shared.

The described process can help to more systematically approach modeling projects in large, heterogenous teams, and to facilitate a clearer understanding of the steps necessary to acquire data with project partners and stakeholders. It is designed to be executed successfully by domain experts from municipal authorities, who are typically not modeling experts.

In further research steps the process will be utilized, evaluated, and extended in additional projects with municical stakeholders, e.g. the EU-H2020 projects "FORESEE - Future proofing strategies for resilient transport networks against extreme events" (see [11]) and "ARCH - Advancing Resilience of Historic Areas Against Climate-related and Other Hazards" (see [2]).

\section{Acknowledgement}

The authors thank their partners in the ARCH and RESIN consortia for their valuable contributions during the development and test process. This paper is based in part upon work in the framework of the European projects "ARCH - Advancing Resilience of Historic Areas Against Climate-related and Other Hazards" and "RESIN - Climate Resilient Cities and Infrastructures".

This project has received funding from the European Union's Horizon 2020 research and innovation programme under grant agreements no. 653,522 and 820,999 . The sole responsibility for the content of this publication lies with the authors. It does not necessarily represent the opinion of the European Union. Neither the EASME nor the European Commission are responsible for any use that may be made of the information contained therein.

\section{References}

[1] Armitage DR, Plummer R, Berkes F, Arthur RI, Charles AT, Davidson-Hunt IJ, Diduck AP, Doubleday NC, Johnson DS, Marschke M, McConney P, Pinkerton EW, Wollenberg EK. Adaptive co-management for social-ecological complexity. In: Frontiers in Ecology and the Environment, Vol. 7, 2009, pp. 95-102.

[2] ARCH - Advancing Resilience of Historic Areas Against Climate-related and Other Hazards, http://www.savingculturalheritage.eu/, accessed on Sept 30, 2019.

[3] Banks J, Carson JS, Nelson BL, Nicol DM. DiscreteEvent System Simulation. Pearson, 2010.

[4] Bengtsson N, Shao G, Johansson B, Leong S, Skoogh A, Mclean C. Input data management methodology for discrete event simulation. In: Proceedings of the 2009 Winter Simulation Conference, 2009, pp. 1335-1344.

[5] Carson JS. Introduction to Modeling and Simulation. In:Proceedings of the 2005 Winter Simulation Conference, 2005, pp. 16-23.

[6] Cash D, Adger WN, Berkes F, Garden P, Lebel P, Olsson P, Pritchard L, Young OR. Scale and cross-scale dynamics. In: Ecology and Society, Vol. 11, 2006.

[7] Chapman E, Hanania S, Connelly A, Carter J. Deliverable D4.2 Developing the RESIN tools. EU H2020 Project RESIN, Freiburg, Germany, 2018.

[8] Cheng R. History of Input Modeling. In: Proceedings of 2017 Winter Simulation Conference, 2017, pp. 181201. 
[9] Dumas M, ter Hofstede A. UML Activity Diagrams as a Workflow Specification Language. In: Proceedings of the 4th Conference on the Unified Modeling Language, 2001, pp. 76-90.

[10] EU General Data Protection Regulation, https://www.eugdpr.org/, accessed Jan 08, 2019.

[11] FORESEE - Future proofing strategies for resilient transport networks against extreme events, https://ec.europa.eu/inea/en/horizon2020/projects/h2020-transport/infrastructure/foresee, accessed Dec 05, 2018.

[12] German Federal Ministry for Economic Cooperation and Development: The Vulnerability Sourcebook. Concept and guidelines for standardised vulnerability assessments. Deutsche Gesellschaft für Internationale Zusammenarbeit, Bonn and Eschborn, Germany, 2014.

[13] Lemos MC, Morehouse BJ. The co-production of science and policy in integrated climate assessments. In: Global Environmental Change, Vol 15, 2005, pp. 5768.

[14] Lückerath D, Bogen M, Rome E, Sojeva B, Ullrich O, Worst R, Xie J. The RESIN climate change adaptation project and its simple modeling approach for riskoriented vulnerability assessment. In: Simulation Notes Europe, Vol. 28, No. 2, 2018, pp. 49-54.

[15] Lückerath D, Dumonteil M. Deliverable D2.5 Applying IVAVIA, a quantitative risk-based vulnerability assessment methodology. EU H2020 Project RESIN, Sankt Augustin, Germany, 2017.

[16] MOVE - Methods for the Improvement of Vulnerability Assessment in Europe, Indicator Database Visualiser, http://www.gi4drr.org/move/, accessed on Oct 25, 2017.

[17] Neves A, Blondel L, Brand K, Hendel Blackford S, Rivas Calvete S, Iancu A, Melica G, Koffi Lefeivre B, Zancanella P, Kona A. The Covenant of Mayors for Climate and Energy Reporting Guidelines, EUR 28160 EN, 2016.

[18] O’Brien K, Eriksen S, Nygaard LP, Schjolden A. Why different interpretations of vulnerability matter in climate change discourses. In: Climate Policy, Vol. 7, 2007, pp.73-88.

[19] OECD: Handbook on constructing composite indicators: methodology and user guide . Paris: OECD Publishing.

[20] Perara T, Liyanage K. Methodology for rapid identification and collection of input data in the simulation of manufacturing systems. In: Simulation Practice and Theory, Vol. 7, No. 7, 2000, pp. 645-656.
[21] Peterson G, De Leo G, Hellmann J, Janssen M, Kinzig A, Malcolm J, O'Brien K, Pope S, Rothman D, Shevliakova E, Tinch R. Uncertainty, Climate Change, and Adaptive Management. In: Ecology and Society, Vol. 1, No. 2, 1997.

[22] Reed MS, Stringer LC, Fazey I, Evely AC, Kruijsen JHJ. Five principles for the practice of knowledge exchange in environmental management. In: Journal of Environmental Management, Vol. 146, 2014, pp. 337345.

[23] RESIN - Climate Resilient Cities and Infrastructures, http://www.resin-cities.eu/, accessed Nov 11, 2018.

[24] Rome E, Bogen M, Lückerath D, Ullrich O, Worst R, Xie J. IVAVIA: Impact and Vulnerability Analysis of Vital Infrastructures and Built-Up Areas. In: Critical Information Infrastructures Security - 13th International Conference, CRITIS 2018, Revised Selected Papers, Springer Security and Cryptology No. 12,260, 2019, pp. 84-97.

[25] Rome E, Bogen M, Worst R, Lückerath D, Sojeva B, Ullrich O, Voss H, Voss N, Xie J. IVAVIA Guideline, Annex to Deliverable D2.3 Realisation and implementation of IVAVIA. EU H2020 Project RESIN, Sankt Augustin, Germany, 2017.

[26] Scheer A-W, Thomas O, Adam O. Process Modeling Using Event-Driven Process Chains (chapter). In: Dumas, M., van der Aalst, M., ter Hofstede, A. (ed.): Process-Aware Information Systems, John Wiley \& Sons, 2005, pp. 119-145.

[27] Skoogh A, Johansson B. Time-consumption analysis of input data activities in discrete event simulation projects. In: Proceedings of the Swedish Production Symposium, Vol. 1, 2007.

[28] Skoogh A, Johansson B, Hanson L. Data Requirements and Representation for Simulation of Energy Consumption in Production Systems. In: Proceedings of CIRP Conference on Manufacturing Systems, Vol. 44, 2011.

[29] Urry J. Sociology Facing Climate Change. In: Sociological Research Online, Vol. 15, 2010, pp. 1-3.

[30] White KP, Ingalls RG. The Basics of Simulation. In: Proceedings of the 2017 Winter Simulation Conference, 2017, pp. 505-519.

[31] Wood MD, Thorne S, Butte G, Linkov I, Kovacs D. Adaptive Management for Climate Change. In: Mental Modeling Approach, Risk, Systems and Decisions. Springer, New York, NY, 2017, pp. 57-67. 